\title{
Short-time-evolved wave functions for solving quantum many-body problems
}

\author{
Orion Ciftja \\ Department of Physics, Prairie View A\&M University, Prairie View, Texas 77446, USA \\ Siu A. Chin \\ Department of Physics, Texas A\&M University, College Station, Texas 77843, USA
}

(Received 30 April 2003; published 6 October 2003)

\begin{abstract}
The exact ground state of a strongly interacting quantum many-body system can be obtained by evolving a trial state with finite overlap with the ground state to infinite imaginary time. In many cases, since the convergence is exponential, the system converges essentially to the exact ground state in a relatively short time. Thus a short-time evolved wave function can be an excellent approximation to the exact ground state. Such a short-time-evolved wave function can be obtained by factorizing, or splitting, the evolution operator to high order. However, for the imaginary time Schrödinger equation, which contains an irreversible diffusion kernel, all coefficients, or time steps, must be positive. (Negative time steps would require evolving the diffusion process backward in time, which is impossible.) Heretofore, only second-order factorization schemes can have all positive coefficients, but without further iterations, these cannot be used to evolve the system long enough to be close to the exact ground state. In this work, we use a newly discovered fourth-order positive factorization scheme which requires knowing both the potential and its gradient. We show that the resulting fourth-order wave function alone, without further iterations, gives an excellent description of strongly interacting quantum systems such as liquid ${ }^{4} \mathrm{He}$, comparable to the best variational results in the literature. This suggests that such a fourth-order wave function can be used to study the ground state of diverse quantum many-body systems, including Bose-Einstein condensates and Fermi systems.
\end{abstract}

DOI: 10.1103/PhysRevB.68.134510

PACS number(s): 67.55.-s, 05.30.Fk, 21.65.+f

We consider a quantum system of $N$ particles with mass $m$ described by the Hamiltonian

$$
H=T+V, \quad T=-\lambda \sum_{i=1}^{N} \nabla_{i}^{2}, \quad V=\sum_{i>j}^{N} v\left(r_{i j}\right),
$$

where $T$ is the kinetic-energy operator, $V$ is a sum of pairwise potentials $v\left(r_{i j}\right)$, and $\lambda=\hbar^{2} /(2 m)$.

In imaginary time $\tau=i t / \hbar$ the many-body time-dependent Schrödinger equation can be written as

$$
-\frac{\partial}{\partial \tau}|\Psi(\tau)\rangle=H|\Psi(\tau)\rangle
$$

with formal solution

$$
|\Psi(\tau)\rangle=e^{-\tau H}|\Phi\rangle, \quad|\Phi\rangle \equiv|\Psi(0)\rangle
$$

In coordinate representation $\Psi(R, \tau)=\langle R \mid \Psi(\tau)\rangle$ $=\left\langle R\left|e^{-\tau H}\right| \Phi\right\rangle$, where $R=\left\{\mathbf{r}_{1} \cdots \mathbf{r}_{N}\right\}$ denotes the set of all particle coordinates. If the initial wave function $|\Phi\rangle$ is expanded in the set of exact eigenfunctions $\left\{\Phi_{n}\right\}$ of the Hamiltonian $H$, then Eq. (3) has the more explicit form

$$
\Psi(R, \tau)=e^{-\tau E_{0}}\left[c_{0} \Phi_{0}+\sum_{n \neq 0}^{+\infty} c_{n} e^{-\tau\left(E_{n}-E_{0}\right)} \Phi_{n}\right]
$$

Assuming the nondegeneracy of the ground state $\left(E_{n}-E_{0}\right.$ $>0 ; n \neq 0)$, the above wave function becomes proportional to the exact ground-state wave function in the limit of infinite imaginary time. A basic strategy is then to start with a good trial wave function and evolve it in imaginary time long enough to damp out all but the exact ground-state wave function.

Since the imaginary time evolution cannot be done exactly, one usually develops a short-time propagator by decomposing $e^{-\tau H}=e^{-\tau(T+V)}$ into exactly solvable parts, and further iterates this short-time propagator to longer time. This is essentially the approach of the diffusion Monte Carlo (DMC) method. ${ }^{1-3}$ The need for iterations introduces the complication of branching, which is the hallmark of diffusion and Green's-function Monte Carlo methods. ${ }^{4}$ Our idea is to develop a short-time propagator via higher-order decomposition that can be applied for a sufficiently long time to project out an excellent approximation to the ground state without iteration.

First- and second-order factorization schemes such as

$$
e^{-\tau(T+V)} \approx\left\{\begin{array}{l}
e^{-\tau T} e^{-\tau V}+O\left(\tau^{2}\right) \\
e^{-(1 / 2) \tau V} e^{-\tau T} e^{-(1 / 2) \tau V}+O\left(\tau^{3}\right)
\end{array}\right.
$$

are well known, but without iterations, they cannot be applied at a sufficiently large value of $\tau$ to get near to the ground state. It is also well known that in the context of symplectic integrators, the short-time-evolution operator can be factorized to arbitrarily high-order in the form ${ }^{5-12}$

$$
e^{-\tau(T+V)}=\prod_{i} e^{-a_{i} \tau T} e^{-b_{i} \tau V}
$$

with coefficients $\left\{a_{i}, b_{i}\right\}$ determined by the required order of accuracy. However, as first proved by Sheng ${ }^{13}$ and later by Suzuki $^{14}$ (using a more geometric argument), beyond second 
order, any factorization of form (6) must contain some negative coefficients in the set $\left\{a_{i}, b_{i}\right\}$. Goldman and Kaper ${ }^{15}$ later proved that any factorization of form (6) must contain at least one negative coefficient for both operators. This means that for decompositions of form (6), one must evolve the system backward in time for some intermediate time steps. This is of little consequence for classical dynamics or real-time quantum dynamics, both of which are time reversible. For the imaginary time Schrödinger equation, whose kinetic-energy operator is the time-irreversible diffusion kernel, this is detrimental. This is because $e^{-a_{i} \tau T}$ $\propto e^{-\left(\mathbf{r}^{\prime}-\mathbf{r}\right)^{2} /\left(2 a_{i} \tau\right)}$ is the diffusion Green's function. For positive $a_{i}$, this kernel can be simulated by Gaussian random walks. If $a_{i}$ were negative, the kernel would be unbound and unnormalizable, with no probabilistic based (Monte Carlo) simulations possible. This is just a mathematical restatement of the physical fact that diffusion is an irreversible process. Positive decomposition coefficients are therefore absolutely essential for solving any evolution equation having an irreversible component, such as the imaginary time Schrödinger equation.

Since both classical and quantum dynamics are time reversible, there is a lack of impetus to search for higher-order factorization schemes with only positive coefficients. While higher-order factorizations of form (6), with negative coefficients, have been studied extensively in the literature, ${ }^{10-12}$ it was only recently that Suzuki ${ }^{16}$ and $\mathrm{Chin}^{17}$ found some fourth-order (but no higher-order) forward time step decomposition schemes. In order to bypass Sheng and Suzuki's proof, one must introduce a higher-order commutator $[V,[T, V]]$ in addition to operators $T$ and $V$ used in Eq. (6). In this work, we use the fourth-order factorization scheme $^{16,17}$ referred to as scheme $A$ :

$$
\begin{aligned}
e^{-\tau(T+V)}= & e^{-(1 / 6) \tau V} e^{-(1 / 2) \tau T} e^{-(2 / 3) \tau \widetilde{V}} e^{-(1 / 2) \tau T} e^{-(1 / 6) \tau V} \\
& +O\left(\tau^{5}\right)
\end{aligned}
$$

with $\widetilde{V}$ given by

$$
\widetilde{V}=V+\frac{\tau^{2}}{48}[V,[T, V]]=V+\frac{\tau^{2}}{48} 2 \lambda \sum_{i=1}^{N}\left|\nabla_{i} V\right|^{2} .
$$

This scheme was also found by Koseloff, ${ }^{18}$ but his coefficient for the double commutator term is incorrect by a factor of 3 too large. For a more detailed discussion of positive factorization schemes and forward symplector integrators, see Ref. 19.

To go from state vectors to coordinate wave functions, we insert complete sets of coordinate states, $\left.1=\int d S \| S\right\rangle\langle S|$, where $S=\left\{\mathbf{s}_{1} \cdots \mathbf{s}_{N}\right\}$ and write, for example, the operator equation (3) in the form

$$
\Psi(R, \tau)=\int d S G(R, S, \tau) \Phi(S),
$$

where Green's function $G(R, S, \tau)$ is given by

$$
G(R, S, \tau)=\left\langle R\left|e^{-\tau H}\right| S\right\rangle .
$$

The intermediate coordinates $S$ are also sometime referred to as "shadow" positions. Each decomposition scheme then corresponds to a specific wave function for the ground state. For instance, the first-order scheme gives the linear wave function

$$
\Psi(R, \tau)=\int d S e^{-C(R-S)^{2}} e^{-\tau V(S)} \Phi(S),
$$

where $(R-S)^{2} \equiv \sum_{i=1}^{N}\left(\mathbf{r}_{i}-\mathbf{s}_{i}\right)^{2}$, and where we have used the fundamental result that the kinetic evolution operator is just the diffusion Green's function,

$$
\left\langle R\left|e^{-\tau T}\right| S\right\rangle \propto e^{-C(R-S)^{2}}, \quad C=\frac{1}{4 \tau \lambda} .
$$

Similarly, the second-order scheme gives the following quadratic wave function:

$$
\Psi(R, \tau)=e^{-(\tau / 2) V(R)} \int d S e^{-C(R-S)^{2}} e^{-(\tau / 2) V(S)} \Phi(S) .
$$

Finally, the fourth-order scheme $A$ produces the following quartic many-body wave function:

$$
\begin{aligned}
\Psi(R, \tau)= & e^{-(\tau / 6) V(R)} \int d S^{\prime} e^{-C^{\prime}\left(R-S^{\prime}\right)^{2}} e^{-(2 \tau / 3) \tilde{V}\left(S^{\prime}\right)} \\
& \times \int d S e^{-C^{\prime}\left(S^{\prime}-S\right)^{2}} e^{-(\tau / 6) V(S)} \Phi(S),
\end{aligned}
$$

now with $C^{\prime}=1 /(2 \tau \lambda)$.

In all these wave functions, there is only a single parameter, the imaginary time $\tau$, that we can vary. All else are fixed by the factorization scheme. If the factorization scheme can accurately reproduce the imaginary time evolution of the wave function, the resulting energy must fall monotonically from the initial energy toward the exact ground-state energy with increasing $\tau$. To the extent that these wave functions are not the exact imaginary time wave function, the energy will eventually rise again. Thus for each wave function there is a optimal $\tau$ where it will be "closest" to the exact ground state.

To test the quality of the above wave functions we use them to describe the ground state of a strongly correlated quantum system of $N^{4} \mathrm{He}$ atoms interacting via a two-body Aziz HFDHE2 potential. ${ }^{20}$ At equilibrium, the system is in a liquid state and has a density of $\rho \sigma^{3}=0.365(\sigma=2.556 \AA)$. The simplest description of the ground state is McMillan's Jastrow wave function

$$
\Phi(R)=\exp \left[-\sum_{i>j}^{N} u\left(r_{i j}\right)\right], u(r)=\frac{1}{2}\left(\frac{b}{r}\right)^{5},
$$

with $b=1.2 \sigma$. We will use this wave function as our initial wave function in all our simulations.

For all three wave functions, the expectation value of the Hamiltonian can be computed from 


$$
E=\frac{\int d R \Psi(R, \tau) H \Psi(R, \tau)}{\int d R|\Psi(R, \tau)|^{2}} .
$$

The iterated wave functions simply require more integration variables. For example, in the case of the linear and quadratic wave function, the above can be expressed as

$$
E=\int d R d S_{L} d S_{R} p\left(R, S_{L}, S_{R}\right) E_{L}\left(R, S_{L}, S_{R}\right),
$$

where $p\left(R, S_{L}, S_{R}\right)$ is the probability density function, $E_{L}\left(R, S_{L}, S_{R}\right)$ is the local energy, and $S_{L, R}$ are the respective left $(L)$, right $(R)$ auxiliary, or shadow, variables. For the quartic wave function, the corresponding expressions for the probability density function and energy expectation value are similar, but with the addition of two more auxiliary shadow variables $S_{L, R}^{\prime}$.

We use the Metropolis Monte Carlo algorithm ${ }^{21}$ to sample the probability density from a $9 N$ - and $15 N$-dimensional configuration space, corresponding to two and four sets of shadow coordinates in the case of linear/quadratic and quartic wave functions, respectively. In these computations, the Metropolis steps are subdivided in two parts. First, one attempts to move real particle coordinates at random inside cubical boxes of side length $\Delta$. Second, analogous attempts are made to move shadow coordinates inside cubical boxes of side length $\Delta_{s h}$. For instance, in the case of the quadratic wave function, we first attempted to move all the $R$ coordinates, then the shadow coordinates $\left\{S_{L}\right\}$ and $\left\{S_{R}\right\}$. The parameters $\Delta$ and $\Delta_{s h}$ were adjusted so that the acceptance ratio for both particle and shadow moves was nearly $50 \%$.

In addition to the ground-state variational energy, we have also computed the radial distribution function $g(r)$, and its Fourier transform, the structure factor $S(k)$. These quantities are spherical averages and have been computed for both the real particles and the shadow coordinates. The radial distribution function is defined by

$$
g(r)=\frac{1}{N \rho} \sum_{i \neq j}^{N}\left\langle\delta\left(\left|\mathbf{r}_{i}-\mathbf{r}_{j}-\mathbf{r}\right|\right)\right\rangle,
$$

where the angular brackets denote an average with respect to $|\Psi(R, \tau)|^{2}$ and $\rho$ is the particle density. The structure factor $S(k)$ is obtained from the average $(1 / N)\left\langle\rho_{-\mathbf{k}} \rho_{\mathbf{k}}\right\rangle$, where $\rho_{\mathbf{k}}$ $=\sum_{j=1}^{N} \exp \left(-i \mathbf{k} \cdot \mathbf{r}_{j}\right)$, a procedure which is only possible on a discrete set of $\mathbf{k}$ values allowed by the periodic boundary conditions.

All simulations presented in this work have been done with $N=108$ atoms of ${ }^{4} \mathrm{He}$ in a cubic box with periodic boundary conditions. To enforce periodicity all correlations smoothly go to zero at a cutoff distance, $r_{c}=L / 2$, equal to half the side of the simulation box according to the replacement

$$
f(r) \rightarrow f(r)+f\left(2 r_{c}-r\right)-2 f\left(r_{c}\right) .
$$

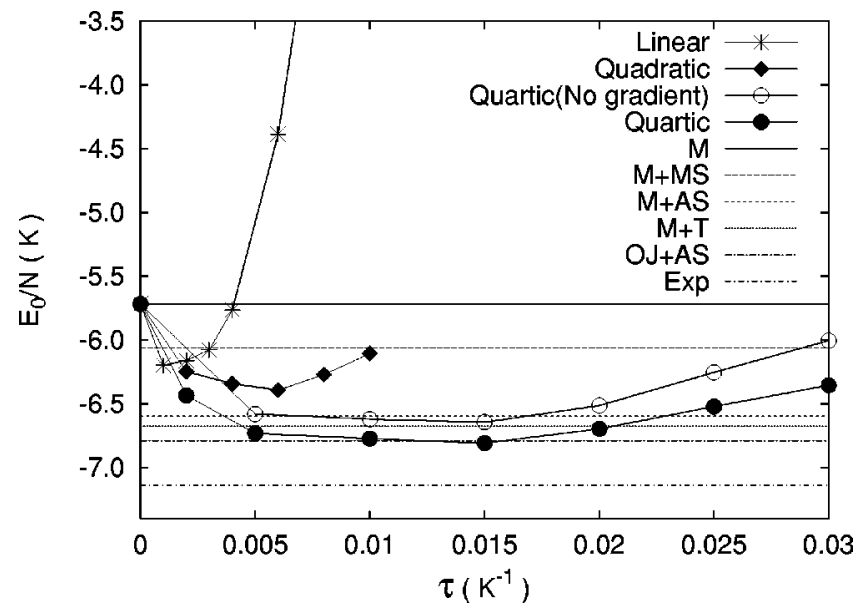

FIG. 1. The ground-state energy per particle in kelvin for ${ }^{4} \mathrm{He}$ at the experimental equilibrium density $\left(\rho \sigma^{3}=0.365\right)$ using the Aziz HFDHE2 potential as a function of the parameter $\tau$. Monte Carlo results from using various short-time-evolved wave functions are as indicated. All simulations have been done for $N=108$ particles. M indicates a McMillan wave-function energy. $\mathrm{M}+\mathrm{MS}, \mathrm{M}+\mathrm{AS}, \mathrm{M}$ $+\mathrm{T}, \mathrm{OJ}+\mathrm{AS}$ refers to various variational Monte Carlo (VMC) results in the literature, see text for details.

In Fig. 1 we show the equilibrium energy per particle for liquid ${ }^{4} \mathrm{He}$ for various short-time-evolved wave functions as function of the imaginary time parameter $\tau$. Other results from literature are also indicated for comparison: M+MS is the energy obtained by a shadow wave function having a pure repulsive McMillan (M) pseudopotential ${ }^{22}$ of fifth power-law form for both particles and shadows. ${ }^{23} \mathrm{M}+\mathrm{AS}$ is the energy obtained by a shadow wave function with an attractive shadow-shadow pseudopotential of scaled Aziz HFDHE2 potential (AS) form. ${ }^{24} \mathrm{M}+\mathrm{T}$ is the McMillan wave function with triplet $(\mathrm{T})$ correlations. ${ }^{25} \mathrm{OJ}+\mathrm{AS}$ refers to a shadow wave function with an optimized Jastrow particleparticle pseudopotential (OJ) and scaled Aziz HFDHE2 shadow-shadow pseudopotential (AS). ${ }^{24}$ GFMC is the Green's-Function Monte Carlo calculations with Mcmillan form for importance and starting function. ${ }^{4}$ The experimental value is taken from Roach et al. ${ }^{26}$

As expected, each of our factorized wave functions reaches an energy minimum with increasing value of $\tau$. The flatness and depth of the energy minimum improve markedly with the order of the wave function. The linear wave function has a shallow and narrow minimum at $\tau=0.002$ and only improves upon McMillan's result $(\tau=0)$ by $\approx 0.3 \mathrm{~K}$. The minimum of the quadratic wave function is much better at $\tau=0.006$ with a value of $-6.393 \mathrm{~K}$. The quartic wavefunction's energy minimum extends further out to $\tau=0.015$ attaining $-6.809 \mathrm{~K}$, which is lower than all existing variational Monte Carlo (VMC) calculations that we are aware of. To demonstrate the necessity of the gradient term, we have also plotted results obtained from Eq. (7) without the gradient term in the potential. In the present case, gradient term is responsible for $\approx 50 \%$ of the improvement from that of the quadratic wave function.

To give a quantitative comparison, we summarize various ground-state equilibrium energies for ${ }^{4} \mathrm{He}$ in Table I.

In Fig. 2 we show the equilibrium pair distribution func- 
TABLE I. Energies of liquid ${ }^{4} \mathrm{He}$ at the experimental equilibrium density $\left(\rho \sigma^{3}=0.365 ; \sigma=2.556 \AA\right)$ and at zero temperature. VMC indicates a variational Monte Carlo calculation with the indicated wave function. All simulations use the Aziz HFDHE2 potential and have been performed for systems of $N=108$ particles. The $\mathrm{M}+\mathrm{MS}$ results are taken from Vitiello et al. (Ref. 23). The M + AS and OJ-AS results are taken from MacFarland et al. (Ref. 24), The GFMC results are taken from Kalos et al. (Ref. 4). The experimental data are taken from Roach et al. (Ref. 26). The energies are given in kelvin per particle.

\begin{tabular}{lcc}
\hline \hline Method & Trial function & Energy (K) \\
\hline VMC & M+MS & $-6.061 \pm 0.025$ \\
VMC & M+AS & $-6.599 \pm 0.034$ \\
VMC & OJ+AS & $-6.789 \pm 0.023$ \\
VMC & Linear & $-6.144 \pm 0.092$ \\
VMC & Quadratic & $-6.393 \pm 0.021$ \\
VMC & No Grad & $-6.644 \pm 0.026$ \\
VMC & Quartic & $-6.809 \pm 0.017$ \\
GFMC & & $-7.120 \pm 0.024$ \\
Experiment & & -7.140 \\
\hline \hline
\end{tabular}

tion $g(r)$ for ${ }^{4} \mathrm{He}$ as obtained from the quartic wave function. This $g(r)$ is compared with the respective $g(r)$ obtained from the M+AS shadow wave function and the experimental one of Svensson et al. ${ }^{27}$ obtained by neutron diffraction at saturated vapor pressure at $T=1.0 \mathrm{~K}$. It is known ${ }^{24}$ that the $\mathrm{M}+\mathrm{AS}$ curve differs from the experimental one because it predicts a diminished nearest-neighbor maximum and the entire curve is shifted by about $0.1 \AA$ to larger values of $r$ compared to the experimental results. The pair distribution function that we obtained is in excellent agreement with the experimental one.

In Fig. 3 we show $S(k)$ at equilibrium density $\rho \sigma^{3}$

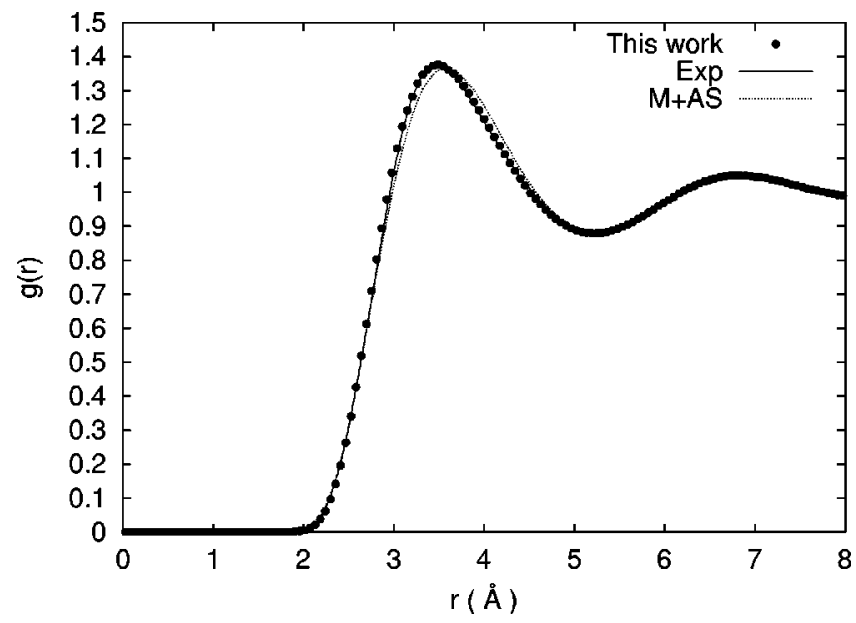

FIG. 2. The pair distribution function for liquid ${ }^{4} \mathrm{He}$ at the equilibrium density $\rho \sigma^{3}=0.365$ after a VMC simulations with $N$ $=108$ particles. The filled circles show the $g(r)$ of this work that is compared with the respective $g(r)$ obtained from the M+AS wave function (dotted line) and the experimental $g(r)$ as reported by Svensson et al. (Ref. 27) (solid line) obtained at saturated vapor pressure at a temperature $T=1.0 \mathrm{~K}$.

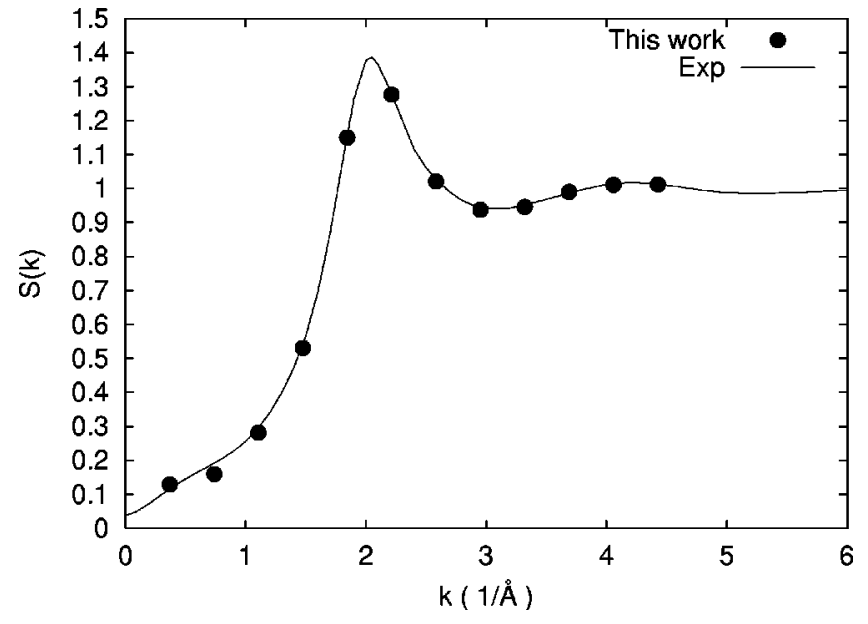

FIG. 3. Static structure factor $S(k)$ of liquid ${ }^{4} \mathrm{He}$ at equilibrium density $\rho \sigma^{3}=0.365$. The filled circles show our results for $S(k)$ obtained from the formula $S(k)=(1 / N)\left\langle\rho_{-\mathbf{k}} \rho_{\mathbf{k}}\right\rangle$. The solid line denotes the experimental results reported by Svensson and co-workers (Ref. 27) obtained at saturated vapor pressure by means of neutron diffraction at temperature $T=1.0 \mathrm{~K}$.

$=0.365$ as obtained from the quartic wave function. The experimental $S(k)$ shown in this figure is the result reported by Svensson $e t a l .{ }^{27}$ The overall agreement between our shorttime-evolved structure factor with experiment is excellent except at small $k$. This is not unexpected because our imaginary time is still rather short for the wave function to develop the necessary long-range correlation to produce the linear behavior ${ }^{28}$ of $S(k)$ observed in bulk ${ }^{4} \mathrm{He}$.

In this work, based on recent findings on forward time steps decomposition schemes, we have implemented a fourth-order short-time-evolved wave function for describing the ground state of strongly interacting quantum systems. Our approach is systematic, free of arbitrary parameters, and can be applied to any general quantum many-body problem. In the case of liquid ${ }^{4} \mathrm{He}$, we have produced ground-state energy and structure results better than any existing VMC calculations, but without the use of complicated branching processes as in DMC or GFMC. Since the antisymmetric requirement on fermion wave functions can be more easily implemented on the variational level, our quartic wave functions may be of great utility in studying Fermi systems.

Our second-order wave function is similar in structure to the class of shadow wave functions, ${ }^{29}$ except that our wave function follows directly from the second-order factorization scheme without any particular adjustment of pseudopotential or scale functions. Our use of a positive factorization scheme to produce a much improved fourth-order wave function demonstrates that there is a systematic way of improving this class of wave functions by introducing more shadow coordinates. Currently, there is no known sixth-order forward factorization schemes, and hence no sixth-order short-timeevolved wave function is possible.

This work was supported, in part, by the National Science Foundation, Grant Nos. PHY-0100839 and DMS-0310580 to one of the authors (S.A.C.). 
${ }^{1}$ P.J. Reynolds, D.M. Ceperley, B.J. Alder, and W.A. Lester, J. Chem. Phys. 77, 5593 (1982).

${ }^{2}$ J.W. Moskowitz, K.E. Schmidt, M.E. Lee, and M.H. Kalos, J. Chem. Phys. 77, 349 (1982).

${ }^{3}$ Siu A. Chin, Phys. Rev. A 42, 6991 (1990).

${ }^{4}$ M.H. Kalos, M.A. Lee, P.A. Whitlock, and G.V. Chester, Phys. Rev. B 24, 115 (1981).

${ }^{5}$ E. Forest and R.D. Ruth, Physica D 43, 105 (1990).

${ }^{6}$ M. Creutz and A. Gocksch, Phys. Rev. Lett. 63, 9 (1989).

${ }^{7}$ J. Candy and W. Rozmus, J. Comput. Phys. 92, 230 (1991).

${ }^{8}$ M. Suzuki, Phys. Lett. A 146, 319 (1990); 165, 387 (1992).

${ }^{9}$ H. Yoshida, Phys. Lett. A 150, 262 (1990).

${ }^{10}$ R.I. McLaclan and P. Atela, Nonlinearity 5, 542 (1991).

${ }^{11}$ R.I. McLachlan, Annu. Stat. Suppl. Soc. Secur Bull. 16, 151 (1995).

${ }^{12}$ P. V. Koseleff, Integration Algorithms and Classical Mechanics, Fields Institute Communications, Vol. 10 (American Mathematical Society, Providence, RI, 1996), p. 103.

${ }^{13}$ Q. Sheng, IMA J. Numer. Anal. 9, 199 (1989).

${ }^{14}$ M. Suzuki, J. Math. Phys. 32, 400 (1991).

${ }^{15}$ D. Goldman and T.J. Kaper, SIAM (Soc. Ind. Appl. Math.) J. Numer. Anal. 33, 349 (1996).

${ }^{16}$ M. Suzuki, Computer Simulation Studies in Condensed Matter Physics VIII, edited by D. Landau, K. Mon, and H. Shuttler
(Springler, Berlin, 1996).

${ }^{17}$ S.A. Chin, Phys. Lett. A 226, 344 (1997).

${ }^{18}$ P.V. Koseleff, in Applied Algebra, Algebraic Algorithms and Error-Correcting Codes, Lecture Notes in Computational Science Vol. 673 (Springer, Berlin, 1993), pp. 213-230.

${ }^{19}$ Siu A. Chin and C.R. Chen, astro-ph/0304223 (unpublished).

${ }^{20}$ R.A. Aziz, V.P.S. Nain, J.S. Carley, W.L. Taylor, and G.T. McConville, J. Chem. Phys. 70, 4330 (1979).

${ }^{21}$ N. Metropolis, A.W. Rosenbluth, M.N. Rosenbluth, A.M. Teller, and E. Teller, J. Chem. Phys. 21, 1087 (1953).

${ }^{22}$ W.L. McMillan, Phys. Rev. 138A, 442 (1965).

${ }^{23}$ S.A. Vitiello, K.J. Runge, G.V. Chester, and M.H. Kalos, Phys. Rev. B 42, 228 (1990).

${ }^{24}$ T. MacFarland, S.A. Vitiello, L. Reatto, G.V. Chester, and M.H. Kalos, Phys. Rev. B 50, 13577 (1994).

${ }^{25}$ K.E. Schmidt, M.H. Kalos, M.A. Lee, and G.V. Chester, Phys. Rev. Lett. 45, 573 (1980).

${ }^{26}$ P.T. Roach, J.B. Ketterson, and C.W. Woo, Phys. Rev. A 2, 543 (1970).

${ }^{27}$ E.C. Svensson, V.F. Sears, A.D.B. Woods, and P. Martel, Phys. Rev. B 21, 3638 (1980).

${ }^{28}$ L. Reatto and G.V. Chester, Phys. Rev. 155, 88 (1967).

${ }^{29}$ S. Vitiello, K. Runge, and M.H. Kalos, Phys. Rev. Lett. 60, 1970 (1988). 